\title{
Neonatal cerebral sinovenous thrombosis: Two cases, two different gene polymorphisms and risk factors
}

\author{
Özden Turan¹, Deniz Anuk-İnce ${ }^{1}$, Lale Olcay², Taner Sezer ${ }^{3}$, Kaan Gülleroğlu, \\ Zerrin Yılmaz-Çelik ${ }^{5}$, Ayşe Ecevit ${ }^{1}$ \\ Divisions of ${ }^{1}$ Neonatology, ${ }^{2}$ Pediatric Hematology, ${ }^{3}$ Pediatric Neurology, ${ }^{4}$ Pediatric Nephrology and ${ }^{5}$ Department of Medical \\ Genetics, Baskent University Faculty of Medicine, Ankara, Turkey. E-mail: drozdentr@yahoo.com \\ Received: 8th August 2016, Revised 29th August 2016, Accepted: 30th September 2016
}

\begin{abstract}
SUMMARY: Turan Ö, Anuk-İnce D, Olcay L, Sezer T, Gülleroğlu K, YılmazÇelik Z, Ecevit A. Neonatal cerebral sinovenous thrombosis: Two cases, two different gene polymorphisms and risk factors. Turk J Pediatr 2017; 59: 71-75.

Cerebral sinovenous thrombosis (CSVT) is a rare disease in the neonatal period and also the greatest risk of neonatal mortality and morbidity. In this report, we presented two cases with CSVT and different risk factors. One of these cases had methylenetetrahydrofolate reductase (MTHFR) C677T homozygous polymorphism and the other case had both MTHFR A1298C homozygous polymorphism, plasminogen activator inhibitor-1 (PAI-1) 4G/ $5 \mathrm{G}$ polymorphism and elevated lipoprotein a. Early diagnosis and prompt initiation of therapy of neonatal CSVT may prevent neonatal mortality and poor long-term neurodevelopmental outcomes.
\end{abstract}

Key words: anticoagulation, cerebral sinovenous thrombosis, newborn.

Cerebral sinovenous thrombosis (CSVT) is a rare disease in the neonatal period and also the greatest risk of neonatal mortality and morbidity. Symptoms are usually nonspecific and may present as seizures, respiratory distress, lethargy, jitteriness, dilated scalp veins, poor feeding, hemiparesis and hemiplegia. Seizures are the most common neurologic symptom in neonatal CSVT, while symptoms related to intracranial hypertension and focal neurologic signs are more frequent in children. In this report, we presented two cases with CSVT and different risk factors. One of these cases had methylenetetrahydrofolate reductase (MTHFR) C677T homozygous polymorphism and the other case had both MTHFR A1298C homozygous polymorphism, plasminogen activator inhibitor-1 (PAI-1) 4G/ $5 \mathrm{G}$ polymorphism and elevated lipoprotein $\mathrm{a}$.

\section{Case Reports}

Case 1

A 41-year-old, gravida 1 mother delivered a male baby at a gestational age of 33 weeks by cesarean section, with a birth weight (BW) of 2,240 g. Apgar scores were 8 and 9 at 1 and 5 minutes, respectively. There was no history of maternal disease in uncomplicated pregnancy. Parents were not consanguineous. Transposition of the great arteries (TGA) was detected at the 26th week on the fetal echocardiography. His physical examination was normal except for pansystolic heart murmur on mesocardiac focus. He was intubated and started intravenous prostaglandin infusion. Echocardiography revealed TGA, muscular ventricular septal defect (VSD) and patency of ductus arteriosus (PDA). Septostomy was performed on the second day of life. On the postnatal third day, the patient had focal seizures on the left arm. He was given phenobarbital and no seizure was observed afterwards. Complete blood count, biochemical parameters, C- reactive protein level, EEG scan, transfontanelle and abdominal ultrasonography were normal. Protein $\mathrm{C}$, total protein S, antithrombin III, factor I, VII, VIII, XII and von Willebrand factor levels were normal. The lupus anticoagulant, anticardiolipin antibody, anti dsDNA and ANA levels were negative. The magnetic resonance imaging (MRI) and MRI venography scan of the brain showed thrombotic lesions in the superior sagittal sinus and the both transverse sinuses, in addition to few and very tiny spots of 
Table I. Diagnostic Tests for Thrombophilia of Two Cases

\begin{tabular}{lll}
\hline Diagnostic Tests & Case 1 & Case 2 \\
\hline Homocysteine, $\mu$ mol/L (normal: 5.46-14) & 6.47 & 7.7 \\
Vitamin B12, pg/ml (normal: 138-652) & 405 & 209 \\
Folic acid, ng/ml (normal: 3.1-20.5) & 13.7 & 12 \\
Factor V Leiden & Homozygous normal & Homozygous normal \\
Prothrombin 20210A & Homozygous normal & Homozygous normal \\
MTHFR C677T polymorphism & Homozygous mutant & Homozygous normal \\
MTHFR A1298C polymorphism & Homozygous normal & Homozygous mutant \\
PAI-1 (4G/5G) polymorphism & $5 \mathrm{G} / 5 \mathrm{G}$ & $4 \mathrm{G} / 5 \mathrm{G}$ \\
d-dimer, ug/ml (normal: <0.5) & 3.84 & 0.36 \\
\hline
\end{tabular}

MTHFR: methylenetetrahydrofolate reductase, PAI: plasminogen activator inhibitor-1

hemorrhage in the cortico medullary junction and diffuse edema in the periventricular white matter (Fig. 1). Although the dimensions of the hemorrhagic spots were very small (in milimetric dimensions) and not significant. He was started on subcutaneous low molecular weight heparin (LMWH), $150 \mathrm{U} / \mathrm{kg}$, twice daily adjusting the dose to keep the anti factor Xa level between 0.5-1.0 U/ml. He had homozygous MTHFR C677T polymorphism. Homocysteine level was normal. All diagnostic laboratory studies for thrombophilia were presented in Table I.

The control brain MRI taken after 2 weeks showed a minimal residual thrombosis in the left transverse sinus and disappearence of tiny hemorrhagic spots. He underwent TGA correction (Jaten) operation on the 29th day of life. Since brain MRI scan taken in the 3rd month was normal, LMWH and anticonvulsant were stopped on the third month of life. Prophylactic treatment was not given. He is now 11 months old without any neurologic deficits.

\section{Case 2}

A 20-year-old, gravida 1 mother delivered a male baby at a gestational age of 38 weeks by cesarean section, with a BW of 3,700 g. Apgar scores were unknown. The patient was discharged from another hospital on the second day of life. There was no history of prenatal problems and maternal disease. Parents were not consanguineous. When he was admitted to our hospital on the eighth day of life, he was dehydrated (weight loss 29\%), had lethargy, anuria and poor neonatal reflexes. He could not be fed by breast milk or other formulated feedings properly. The initial laboratory findings were as follows: blood sodium $186 \mathrm{mEq} / \mathrm{L}$, potassium $7.4 \mathrm{mEq} / \mathrm{L}$, creatinine $4.8 \mathrm{mg} /$ $\mathrm{dl}$, hemoglobin $16 \mathrm{~g} / \mathrm{dl}$, haematocrit 54\%, MCV108/fL, WBC 12,700/ $\mathrm{mm}^{3}$, platelets $435,000 / \mathrm{mm}^{3}$. Protein C, total protein $\mathrm{S}$, antithrombin III, factor I, VII, VIII, XII and von Willebrand factor levels were normal. The lupus anticoagulant, anticardiolipin antibody, anti dsDNA and ANA levels were negative. Transfontanelle ultrasonography was normal. Renal ultrasonography revealed medullary nephrocalcinosis. The MRI and MRI venography scan of the brain showed thrombotic lesions in both transverse sinuses (Fig. 2).

He was started on subcutaneous LMWH as $150 \mathrm{U} / \mathrm{kg}$ twice daily, adjusting the dose to keep the anti factor Xa level between 0.51.0. The brain computed tomography (CT) taken on the 3rd day of LMWH treatment showed no hemorrhage. He had homozygous MTHFR A1298C polymorphism and PAI 4G/5G polymorphism, and elevated lipoprotein a. Other diagnostic tests for thrombophilia were presented in Table I. The brain MRI taken after 8 weeks of therapy revealed that the thrombosis had resolved completely. Total duration of acute anticoagulation therapy was 2 months and prophylactic treatment was not given. Due to mild vitamin B12 deficiency, vitamin B12 therapy was started. Neurologic signs were irrelevant to vitamin B12 deficiency. $\mathrm{He}$ is now 6 months old without any neurologic deficits. Informed consent was received from the families.

\section{Discussion}

Cerebral sinovenous thrombosis is an 


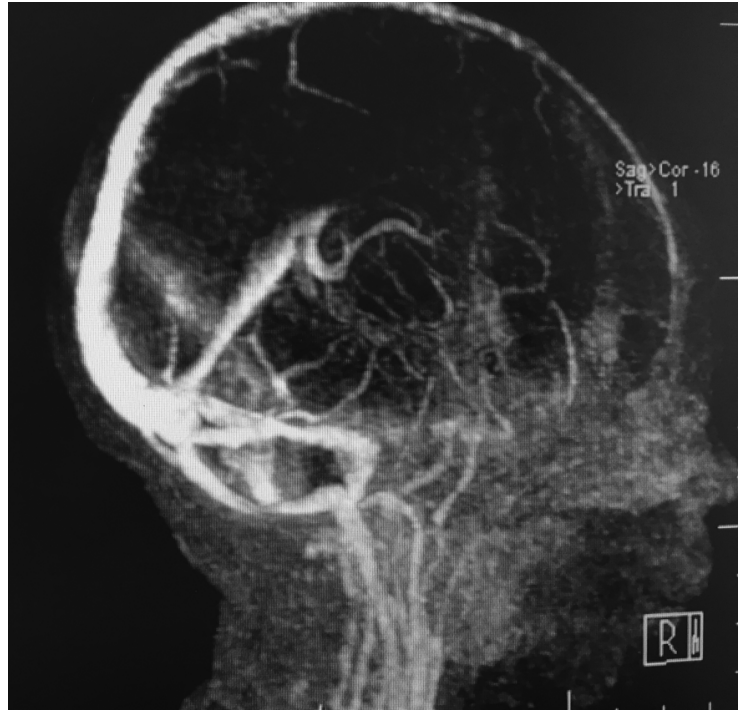

Fig. 1. The magnetic resonance imaging (MRI) venography scan of the brain showed thrombotic lesions in the superior sagittal sinus and the both transverse sinuses

uncommon disease in children. The true incidence of CSVT is unknown in neonates and children. Although likely underestimated, CSVT occurs in about one of 100,000 children per year and approximately half of the CSVT in children occur in the neonatal period or in early infancy. Important risk factors causing CSVT in newborns include congenital heart disease (TGA), perinatal hypoxic ischemic encephalopathy, meconium aspiration syndrome, Apgar score $<7$ at 5 minute, intubation at birth, postnatal sepsis, meningitis, dehydration, polycythemia, pneumonia, ECMO treatment, disseminated intravascular coagulation, congenital diaphragmatic hernia, placental premature rupture of membranes, ablatio plasenta, chorioamnionitis and maternal gestational diabetes mellitus and preeclampsia, hypertension problems ${ }^{1-3}$.

Other risk factors are presence of prothrombotic states like factor V G1691A (Leiden) mutation, prothrombin G20210A mutation, protein $\mathrm{C}$, protein $\mathrm{S}$ and antithrombin III deficiencies, elevated lipoprotein (a) levels and anticardiolipin antibodies, increased factor VIII level, hyperhomocyteinemia and homozygosity for thermolabile variant of the MTHFR gene, homocystinuria ${ }^{2,4}$. Prothrombotic states were detected in $20 \%$ of neonates and $24-64 \%$ of children with CSVT $^{2}$. Herein, we presented two CSVT cases with two different gene

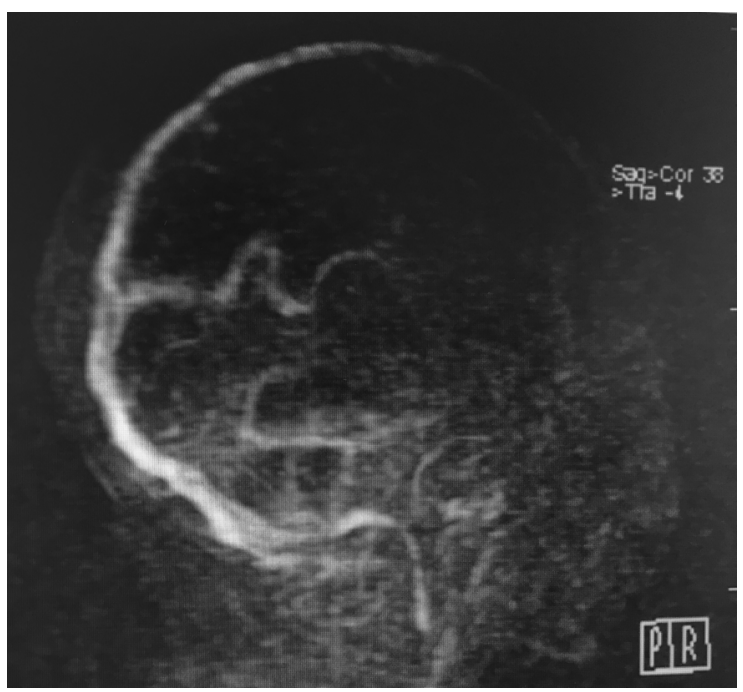

Fig. 2. The magnetic resonance imaging (MRI) venography scan of the brain showed thrombotic lesions in the both transverse sinuses

polymorphisms and risk factors.

C677T polymorphism is the most common mutation in MTHFR polymorphism. Catalano et al. ${ }^{5}$ found that MTHFR A1298C gene heterozygous polymorphism had more severe non-alcoholic fatty liver disease. There has been very rarely reported case of CSVT associated with MTHFR A1298C mutation in the neonatal period. Homozygoous mutation for MTHFR A1298C with CSVT has been previously reported in a newborn infant ${ }^{6}$. In this case, clinical presentation was seizure but had no other risk factors for CSVT. MTHFR gene mutation analysis noted a positive C677T homozygous mutation and MTHFR A1298C homozygous mutation in our patients. In addition to MTHFR mutations, our first case had congenital heart disease and post catheterization procedure for septostomy. Our second case had hypernatremic dehydration, PAI 4G/5G polymorphism, and elevated lipoprotein a levels which is also risk factor for arterial thrombosis ${ }^{7}$. While the first patient had three, the second patient had four risk factors for venous thrombosis. Hence, at least one risk factor has been identified in more than $90 \%$ of patients in children with CSVT. Lolli et al. ${ }^{7}$ reported that no predisposing factor was significantly higher in newborn infants than older children (36\% and less than $5 \%$, respectively). 
PAI-1 inhibits the activity of urokinase plasminogen activator, which plays an essential role for the cleavage of plasminogen to plasmin. PAI $4 \mathrm{G} / 5 \mathrm{G}$ polymorphism is a common polymorphism in the promoter region of the PAI1 gene. This polymorphism is associated with a slightly increased risk of thromboembolism. Our second case is the first reported neonatal CSVT with homozygotic MTHFR A1298C and PAI 4G/5G polymorphism. Onrat et al. ${ }^{8}$ also reported that the PAI $4 \mathrm{G} / 5 \mathrm{G}$ polymorphism is associated with myocardial infarction in patients before the age of 45 .

Hyperhomocysteinemia is a common result of MTHFR polymorphism ${ }^{9}$. MTHFR polymorphism is not included in the guidelines of thrombophilia among laboratory tests. However, new data suggest that only homocysteinemia or thermolabile variant of MTHFR should be investigated ${ }^{1,10}$.

However, stroke in both C677T and MTHFR A1298C mutations were reported not to be associated with hyperhomocysteinemia. This was attributed to that single time-point measurements may not be enough to represent overall homocysteine abnormalities and homocysteine levels fluctuated with time ${ }^{9,11}$. Therefore, normal levels of homocysteine in single measurements does not rule out hyperhomocysteinemia in our patients.

The best form of treatment for neonatal CSVT is unknown. Although not based on clinical trials, unfractionated heparin or $\mathrm{LMWH}$ administration is therefore advised in patients with SVT for a minimum period of 6 weeks and no longer than 3 months. At the present time, anticoagulant therapy is safe in neonatal CSVT cases $^{12}$.

A meta-analysis showed that mortality was significantly reduced in children/neonates who received anticoagulation as $1.8 \%$ vs $10 \%$. Anticoagulation seemed also to predict reduction in thrombosis and total or partial sinus recanalization, both associated with a reduced risk of a new infarct and an improved clinical outcome ${ }^{13}$. Anticoagulation therapy is less frequently used in neonates. Two determinants of unfavourable outcome as death or sequelae in children with CSVT were reported as neonatal age and absence of anticoagulation therapy ${ }^{13}$. Thorell et al. ${ }^{14}$ showed heparin treatment reduced mortality and severe disability with no increase in risk of intracranial hemorrhage. In the Canadian registry of CSVT over one third of newborns with CSVT received anticoagulant medications without major bleeding or extension of the thrombus ${ }^{12}$. In our patients, we administered LMWH since Case 2 did not have hemorrhage, and Case 1 did not have significant hemorrhage being only as few and very tiny spots of hemorrhage. We did not experience any hematological or neurological complications with LMWH.

Abnormal neurodevelopmental outcomes have been revealed in $40 \%$ to $80 \%$. The spectrum of neurological disabilities reported in previous studies also varied (developmental delay, epilepsy, cerabral palsy, hydrocephalus) but is suggestive of global impairment of function with more severe impairments 3,15-17. Gentilomo et al. ${ }^{17}$ reported that neurological sequelae were significantly higher in prothrombotic abnormalities group compared to the without thrombophilic disease. Bektaş et al. ${ }^{15}$ showed that newborns who did not receive anticoagulant therapy were often worse neurologic outcome.

Cerebral sinovenous thrombosis is a multifactorial and life threatening disease. Treatment of underlying causes like sepsis, dehydration, congenital heart disease is very important in protrombotic disorder. Early diagnosis and prompt initiation of therapy of neonatal CSVT may prevent neonatal mortality and poor long-term neurodevelopmental outcomes.

\section{REFERENCES}

1. Roach ES, Golomb MR, Adams R, et al. American Heart Association Stroke Council; Council on Cardiovascular Disease in the Young. Management of stroke in infants and children: a scientific statement from a Special Writing Group of the American Heart Association Stroke Council and the Council on Cardiovascular Disease in the Young. Stroke 2010; 39: 2644-2691.

2. Dlamini N, Billinghurst L, Kirkham FJ. Cerebral venous sinus (sinovenous) thrombosis in children. Neurosurg Clin N Am 2010; 21: 511-527.

3. deVeber G, Andrew M, Adams C, et al. Canadian Pediatric Ischemic Stroke Study Group. Cerebral sinovenous thrombosis in children. $\mathrm{N}$ Engl J Med 2001; 9: 417-423.

4. Heller C, Heinecke A, Junker R, et al. Childhood Stroke Study Group. Cerebral venous thrombosis in children: a multifactorial origin. Circulation 2003; 108: 1362-1367. 
5. Catalano D, Trovato GM, Ragusa A, et al. Non-alcoholic fatty liver disease (NAFLD) and MTHFR 1298A > C gene polymorphism. Eur Rev Med Pharmacol Sci 2014; 18: $151-159$

6. Cizmeci MN, Kanburoglu MK, Akelma AZ, et al. Cerebral sinovenous thrombosis associated with MTHFR A1298C mutation in the newborn: a case report. J Thromb Thrombolysis 2013; 35: 279-281.

7. Lolli V, Molinari F, Pruvo JP, Soto Ares G. Radiological and clinical features of cerebral sinovenous thrombosis in newborns and older children. J Neuroradiol 2016; 43: $280-289$

8. Onrat ST, Akci O, Söylemez Z, Onrat E, Avşar A. Prevalence of myocardial infarction polymorphisms in Afyonkarahisar, Western Turkey. Mol Biol Rep 2012; 39: 9257-9264.

9. Rook JL, Nugent DJ, Young G. Pediatric stroke and methylenetetrahydrofolate reductase polymorphisms. An examination of C677T and A1298C mutations. J Pediatr Hematol Oncol 2005; 27: 590-593.

10. Raffini L. Thrombophilia in children: Who to test, how, when, and why? Hematology Am Soc Hematol Educ Program 2008; 228-235.

11. Solomon LR. Cobolamin-responsive disorders in the ambulatory care setting: unreliability of cobalamin, methylmalonic acid, and homocysteine testing. Blood 2005; 105: 978-985.
12. Moharir MD, Shroff M, Stephens D, et al. Anticoagulants in pediatric cerebral sinovenous thrombosis: a safety and outcome study. Ann Neurol 2010; 67: 590-599.

13. Lebas A, Chabrier S, Fluss J, et al. French Society for Paediatric Neurology; European Paediatric Neurology Society. EPNS/SFNP guideline on the anticoagulant treatment of cerebral sinovenous thrombosis in children and neonates. Eur J Paediatr Neurol 2012; 16: 219 228.

14. Thorell SE, Parry-Jones AR, Punter M, Hurford R, Thachil J. Cerebral venous thrombosis-a primer for the haematologist. Blood Rev 2015; 29: 45-50.

15. Bektaş Ö, Teber S, Akar N, et al. Cerebral sinovenous thrombosis in children and neonates: clinical experience, laboratory, treatment, and outcome. Clin Appl Thromb Hemost 2015; 21: 777-782

16. Fitzgerald KC, Williams LS, Garg BP, Carvalho KS, Golomb MR. Cerebral sinovenous thrombosis in the neonate. Arch Neurol 2006; 63: 405-409.

17. Gentilomo C, Franzoi M, Laverda AM, Suppiej A, Battistella PA, Simioni P. Cerebral sinovenous thrombosis in children: thrombophilia and clinical outcome. Thromb Res 2008; 121: 589-591. 